\title{
Caracterización de cyberbullying en el gran Santiago de Chile, en el año 2010
}

\author{
Jorge Varela T. \\ Universidad del Desarrollo, Santiago de Chile \\ J. Carola Pérez. \\ Centro de Estudios Evolutivos e Intervención en el Niño (CEEIN), Universidad del Desarrollo \\ Hermann Schwaderer Z. \\ Fundación Paz Ciudadana \\ Javiera Astudillo. \\ Centro de Estudios Evolutivos e Intervención en el Niño (CEEIN), Universidad del Desarrollo \\ Felipe Lecannelier A. \\ Centro de Estudios Evolutivos e Intervención en el Niño (CEEIN), Universidad del Desarrollo
}

\begin{abstract}
Resumen
El estudio caracteriza el cyberbullying en estudiantes del gran Santiago de Chile (2010). Se aplicó una encuesta de autorreporte (Cuestionario de Experiencias de Internet) a 1.357 estudiantes entre $7^{\circ}$ básico a IV ${ }^{\circ}$ medio. El diseño de muestra fue no probabilístico por cuotas en 32 establecimientos educacionales, ponderados según género, curso y tipo de dependencia. El 50\% fueron hombres, con un promedio de edad 15 años (DS= 1,82 años). Resultados: El 11,4\% reportó haber sido víctima de algún tipo de cyberbullyingy el 12,5\% victimario. No se encontraron diferencias significativas en las víctimas según sexo, curso y tipo de dependencia. Sólo los hombres reportan identificarse en forma más frecuente con los victimarios que las mujeres. Conclusiones: El cyberbullying es un fenómeno existente en la realidad chilena, siendo - mayormente homogéneo en su presentación. Dado esto, se plantean nuevos desafíos y preguntas respecto a sus consecuencias tanto para los estudiantes como para todo el sistema escolar.
\end{abstract}

Palabras Clave: Bullying, tecnologías, prevención.

\section{Cyberbullying prevalence in Santiago of Chile, in 2010}

\begin{abstract}
This study characterizes the cyberbullying among students in Santiago, Chile (2010). A self-report questionnaire (Internet Experiences Questionnaire) was completed by 1,357 students from 7th to 12 th grade. The sample design was non-probability and based on quota sampling. The data were weighted by gender, grade and type of school dependency. Among the respondents $50 \%$ were men, average age 15 years (SD $=1.82$ years). The results show that $11.4 \%$ reported having been the victim of some form of cyberbullying and $12.5 \%$ victimizer. No significant differences were found between the gender, grade or type of school dependency of the victims. In the role of perpetrator, boys reported a higher frequency in compare to girls. We conclude that cyberbullying is a phenomenon that exists in Chilean reality, and it is mostly-homogenous in its presentation. Given this, new challenges and questions regarding the consequences of cyberbullying for students and the educational system are raised.
\end{abstract}

Keywords: Bullying, technologies, prevention. 


\section{Introducción}

\section{Bullying y cyberbullying}

El acoso escolar o bullying tiene efectos negativos en el ajuste social de sus víctimas y agresores. Por ejemplo, ser víctima de bullying se relaciona con problemas de ajuste psicosocial y problemas psicológicos (Nansel et al., 2001), con una mayor sintomatología depresiva, ansiedad e ideación suicida, e impacta negativamente en la autoestima (Grills \& Ollendick, 2002; Salmon, James \& Smith, 1998). Estos resultados han sido corroborados en estudios longitudinales (Bond, 2001).

Recientemente ha emergido un nuevo formato de este fenómeno conocido como intimidación escolar cibernética o cyberbullying. Esta nueva forma de victimización entre pares involucra el uso de las tecnologías - teléfono celular, envío de SMS, uso de Internet, etc.,- para ejercer daño sobre una(s) víctima(s), que no puede defenderse fácilmente. El cyberbullying - al igual que en el bullying tradicional - se caracteriza por la intencionalidad de ejercer daño, la reiteración de la conducta agresiva, y el desequilibrio de poderes entre la víctima y el agresor (Langos, 2012). Sin embargo, a diferencia de éste, se caracteriza por la invisibilidad del agresor (Spears, Slee, Owens \& Johnson, 2009), el aumento potencial del número de personas que pueden ser espectadores de la agresión y, la re-victimización constante (Wong-Lo, Bullock \& Gable, 2011).

\section{Acceso a tecnologías de la información}

Chile destaca por la difusión y masificación de las tecnologías de la información (TIC's), colocando a los jóvenes del país frente a frente con el fenómeno del cyberbullying. Por ejemplo, la accesibilidad a Internet aumentó de 250.000 usuarios a más de 4,8 millones de usuarios en el período 1997 -2004 (PNUD, 2006).Adicionalmente, respecto al acceso a telefonía celular, la Subsecretaría de Telecomunicaciones de Chile indicaba que ya en el año 2009 existían 3,4 teléfonos celulares por cada hogar, por lo que se puede concluir que alguno de estos teléfonos se encuentra en manos de uno de los hijos/as de dicho hogar. Datos de nivel nacional indican la gran proliferación del uso de las tecnologías entre los adolescentes. Por ejemplo, la mayoría (el 62,7\%) de los adolescentes entre15 a 19 años indican que utilizan diariamente o casi a diario el computador, y un $58,6 \%$ de ellos indica que utilizan diariamente o casi a diario Internet. Este porcentaje varía según el nivel socio-económico (NSE) al que pertenece el joven, donde aquellos con más recursos tendrían un mayor acceso a estas tecnologías. Adicionalmente, los adolescentes de NSE alto utilizan el computador y acceden a Internet preferentemente en su domicilio, en cambio, los adolescentes de NSE más bajos acceden al uso de computadores en establecimientos educacionales e Internet en Cibercafés (INJUV, 2010). En definitiva, los niños(as) y jóvenes chilenos tendrían alto acceso a Internet, una de las TIC's a través del cual se puede desarrollar cyberbullying. De hecho, destaca que los jóvenes utilizan esta tecnología todos los días o varias veces a la semana para realizar las siguientes actividades: Chatear (el 54\%), enviar/ recibir e-mails (el 45\%), usar Facebook (el 45\%) o Fotolog o Blogs (el 32\%), y participar en comunidad virtuales o foros (el 10\%), todas actividades que pueden ser utilizadas para desarrollar cyberbullying (INJUV, 2010).

\section{Prevalencia cyberbullying}

Datos internacionales indican que aproximadamente 1 de cada 5 estudiantes se han involucrado en dinámicas de cyberbullying. Por ejemplo, en Estados Unidos el $19 \%$ de los jóvenes (10 - 17 años) usuarios regulares de Internet estuvieron involucrados en el último año en conductas de cyberbullying, donde el 3\% indicó ser agresores/víctimas, el $4 \%$ indicó ser víctima y el $12 \%$ indicó ser agresor usando Internet hacia otros jóvenes (Ybarra \& Mitchell, 2004). Por su parte, Hinduja y Patchin (2009) reportaron que cerca del $10 \%$ de los estudiantes han sido víctimas de cyberbullying durante el último mes, y sobre un $17 \%$ reportó haber sido víctima de cyberbullying al menos una vez en su vida.

En Reino Unido, una medición nacional señala que un 25\% de los jóvenes (11 a 19 años) ha sufrido algún tipo de cyberbullying (National Children's Home, 2002). En Canadá, Li (2006) evidenció que cerca de un $25 \%$ de los estudiantes ha sido víctima de cyberbullying y un $17 \%$ ha agredido a otros utilizando medios de comunicación electrónicos.

En países hispano-parlantes, específicamente en España, un 5,5\% de los/as estudiantes que se reconocen víctimas, identifica las nuevas tecnologías como instrumento para los abusos que reciben. En el caso de los agresores esporádicos la incidencia es del $4,8 \%$ y agresores sistemáticos del 0,6\% (Defensor del Pueblo, 2007).

En Chile, la Tercera Encuesta Nacional de Violencia en el Ámbito Escolar realizada el año 2009, indicó que el $10,6 \%$ de los estudiantes declara haber sido agredido por medio de internet (blogs, fotolog, etc.). Solo un 6,4\% de los estudiantes se indicó como victimario por medio de internet (Ministerio del Interior \& Adimark Gfk, 2010).

Las variables socio-demográficas de "Edad" y "Sexo" hacen variar estas prevalencias, no obstante, no existe un patrón único (Wade \& Beran, 2011; Smith et al., 2008). Por ejemplo, Li (2006) no encontró diferencias de género en el rol de víctima de cyberbullying, pero sin el caso de los agresores, en una muestra de estudiantes Canadienses. Específicamente, los hombres auto reportaron ser más agresores (el $22,3 \%$ ) que las mujeres (el $11,6 \%$ ), pero los reportes de víctima es similar en ambos sexos (el 25\% y el $25.6 \%$ ). En otro estudio, Ybarra y Mitchell (2007) reportan, que los estudiantes hombres de Estados Unidos tienen tres veces más probabilidades de ser autores frecuentes de intimidación en línea (denominado Internet Harassment). Ahora bien, mayores estudios son necesarios para poder confirmar un 
posible patrón de victimización por género, y su posible rol moderador de cyberbullying.

Adicionalmente, Smith y colaboradores (2008), y Kowalski, Limber, y Agatston, (2012) reportaron diferencias de edad tanto en los roles de agresores y víctimas de cyberbullying en adolescentes (11 a 16 años de edad). Sin embargo, otros estudios no han encontrado esa diferencia. Por ejemplo, Patchin y Hinduja (2006), sobre la base de una muestra de 571 jóvenes usando una encuesta en línea reportó que no hay relaciones estadísticamente significativas considerando la edad como un moderador. Debido a esto mismo, la investigación en torno a este tema todavía requiere mayor desarrollo para poder explicar y entender las diferencias de edad (Kiriakidis \& Kavoura, 2010).

En este marco, el presente estudio tiene por objetivo describir la prevalencia de cyberbullying, tanto en los roles de víctima como agresor (y/o victimario). Considerando que la prevalencia puede variar según variables demográficas se espera conocer si dichas prevalencias varía según sexo, curso (como proxy de edad) y tipo de dependencia de los establecimientos educacionales (Particular, Particular Subvencionado y Municipal). Se consideran específicamente tres estrategias o formas de ejercer cyberbullying: a) Envío de Mensajes de Texto, b) Intimidación por Internet, y c) Agredir con fotografías tomadas sin consentimiento desde un teléfono celular.

\section{Método}

\section{Participantes}

Estudio de tipo descriptivo cuyo universo comprende los escolares que cursaban entre $7^{\circ}$ año básico a IV ${ }^{\circ}$ año medio en el año 2010, en establecimientos educacionales Municipales, Subvencionados y Particulares, de la cuidad de Santiago de Chile. La muestra fue de 1.357 estudiantes, 32 establecimientos educacionales de la Región Metropolitana. La proporción de hombres alcanzó el $50 \%$, con un promedio de edad 15 años (DS $=1,87$ ) en la muestra total. La proporción de la muestra según el curso fue: $7^{\circ}$ año básico el $17 \%$, $8^{\circ}$ año básico el $17 \%, I^{\circ}$ año medio el $19 \%$, II año medio el $17 \%$, III ${ }^{\circ}$ año medio el $16 \%$ y IV ${ }^{\circ}$ año medio el $14 \%$. A su vez, la proporción según el tipo de establecimientos educacionales fue de un $29 \%$ para los establecimientos Municipales, un $55 \%$ para los establecimientos Subvencionados y un $16 \%$ para los establecimientos Particulares. La muestra fue ponderada y es representativa de la población según género, edad de los adolescentes y tipo de dependencia del establecimiento educacional.

\section{Instrumentos}

El instrumento utilizado es una adaptación a la realidad de Chile (Lecannelier et al., 2010) del "Internet Experien- ces Questionnaire”, (Raskauskas \& Stoltz, 2007). Contiene 28 ítems de autorreporte sobre distintas formas de Bullying y Cyberbullying, medidas considerando el último año escolar.

Se considera víctima de cyberbullying a aquellas personas que indican que han sido víctima de acoso escolar -durante el presente año escolar- a través de Mensajes de Texto (¿Has sido víctima de matonaje o abuso escolar por medio de Mensajes de Texto?), de Internet (¿Has sido víctima de matonaje o abuso escolar por alguien que creó un sitio Web acerca de ti, usando tus fotos en línea sin permiso, creando foros o Blogs acerca de ti, creando mail ofensivos y/o posteando en contra tuya?) y de teléfono celular con opción para tomar fotografías (¿Has sido víctima por alguien que tomó fotos tuyas con su teléfono celular sin tu permiso y hace uso de las fotos para molestarte?).

Los indicadores de agresor de cyberbullying se construyeron considerando las respuestas en las cuales los estudiantes reportan el grado de cercanía o parecido de su comportamiento ("bien parecido a mí", "parecido a mí" y "un poco parecido a mí") respecto a la conducta de cyberbullying antes mencionadas.

\section{Procedimiento}

Los estudiantes fueron contactados en los establecimientos educacionales, cuyos Directores aceptaron participar. Los cuestionarios fueron aplicados en la sala de clases, en el horario normal de clases. Al momento de la aplicación, las personas a cargo explicaron a los estudiantes los objetivos del estudio y detallaron en qué consistía su participación, la cual fue de naturaleza voluntaria. Los participantes contestaron en forma anónima el cuestionario de autoreporte "Internet Experiences Questionnaire". El levantamiento de información se llevó a cabo entre el 6 y el 19 de agosto del año 2010.

\section{Análisis de Datos}

Los datos fueron ingresados al programa SPSS 15.0, y se realizó el análisis de los datos. Se estimaron los porcentajes para establecer las prevalencia del fenómeno en su conjunto (cuando los adolescentes reportaban haber sido víctimas o victimarios en - al menos una - de las modalidades de cyberbullying) y en cada una de estas en forma específica: envío de SMS, a través de internet y enviando fotografías a través de celulares. Se establecieron filtros para determinar del universo de estudiantes encuestados, aquellos que tuvieran acceso a las tecnologías de comunicación a través de las cuales se realiza el cyberbullying. Finalmente, se comparó la prevalencia del fenómeno en su conjunto y en cada una de las modalidades de cyberbullying según género, edad (usando curso como proxy) y tipo de dependencia (Particular, Particular Subvencionado y Municipal). La comparación de los porcentajes se realizó utilizando la prueba de diferencia de proporciones. 


\section{Resultados}

Los resultados indican que el $11,4 \%$ de los alumnos que tienen acceso a las tecnologías celular e internet $(N=$ 1309) reportan haber sido víctima de algún tipo de cyberbullying, ya sea a través del envío de mensajes de texto o fotografías por el celular o en Internet. Respecto a los victimarios, es decir, aquellos que realizarían la agresión a través de los medios tecnológicos, el 12,5\% reconoce hacerlo a través de - al menos una- de las formas de ejercer violencia consideradas en el presente estudio. No se encuentran diferencias estadísticamente significativa entre estos porcentajes $(z=0,84 p>0,05)$.

Al analizar en forma específica cada una de las formas o tipos de cyberbullying, los resultados indican que el $3,6 \%$ del total de escolares que cuentan con celular $(\mathrm{N}=$ 1.192) reportan haber sido víctimas de cyberbullying a través del envío de SMS; y el 4,4\% de ellos indican haber realizado bullying a través de esta tecnología. El 7,9\% del total de escolares que cuentan con acceso a internet $(\mathrm{N}=1.050)$ reportan haber sido víctimas de cyberbullying a través del uso de esta tecnología; y el 5,9\% de ellos reportan haber agredido a otros usando internet. Finalmente, al considerar el envío de fotografías a través de celulares $(\mathrm{N}=1.151)$, el $6,6 \%$ reportan haber sido víctimas de cyberbullying y el $7,8 \%$ de ellos reportan haber agredido a otros a través del uso de ésta tecnología. Al comparar los porcentajes del reporte de victimas respecto de los victimarios, no se encuentran diferencias estadísticamente significativa en el envío de SMS $(z=1,04, p>0,05)$, a través de internet $(z=1,81, p>0,05)$ ni enviando fotografías a través de celulares $(z=1,13, p>$ 0,05).

\section{Prevalencia Según Género}

Los resultados indican que los y las jóvenes reportan haber sido víctima de cyberbullying en forma similar ( $p \geq$ ,05), tanto al considerar la intimidación escolar cibernética en su conjunto (Escala Total, Tabla 1) como en cada una de las modalidades a través del cual el cyberbullying es efectuado (envío de SMS, a través de Internet, o enviando fotos a través de internet). La única excepción lo constituye el hecho que las mujeres indican en forma más frecuente que los hombres no haber sido víctimas de cyberbullying a través del envío de fotografías no consensuadas $(z=-4,44$, $p<, 05)$, y estos tienen una tasa de no respuesta mayor que las mujeres $(z=2,15, p<, 05)$.

En cambio, es más frecuente que los hombres reconozcan haber realizado cyberbullying comparado con las mujeres. Así, al considerar la Escala Total, los estudiantes se identifican realizando alguna de las conductas de cyberbullying $(z=2,4, p<, 05)$ en forma más frecuente que las mujeres y estas - por su parte - no se identifican con estas conductas $(z=-3,58, p<, 05)$ en forma más frecuente que los hombres. No obstante, debe mencionarse que la tasa de no respuesta en los hombres es superior a la de las mujeres $(z=2,88, p<, 05)$.

Similar resultados se aprecian al analizar las conductas especificas de agredir a terceros a través de Internet, del envío de SMS y/o el envío de fotos no consensuadas a través del celular (ver Reporte de Victimarios, Tabla 1). Los resultados en las diferentes estrategias de cyberbullying indican que, las mujeres explícitamente indican que no se identifican con la realización de acoso a través de mensajería de texto $(z=-2,56, p<, 05)$ e internet $(z=-4,54, p<, 05)$ en forma más frecuente que los hombres. Asimismo, los hombres reportan

Tabla 1. Prevalencia cyberbullying según Género: Estudiantes víctimas y agresores

\begin{tabular}{|c|c|c|c|c|c|c|c|c|c|}
\hline & & \multicolumn{2}{|c|}{ SMS } & \multicolumn{2}{|c|}{ Internet } & \multicolumn{2}{|c|}{ Fotografías } & \multicolumn{2}{|c|}{ Total } \\
\hline & & $\mathrm{F}^{\mathrm{b}}$ & M & $\mathrm{F}$ & M & $\mathrm{F}$ & M & $\mathrm{F}$ & M \\
\hline \multirow{3}{*}{ 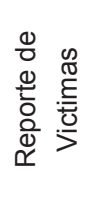 } & $\mathrm{SI}$ & 4,4 & 2,8 & 9,4 & 6,5 & 5,9 & 7,3 & 13,6 & 11,4 \\
\hline & $\mathrm{NO}$ & 94,6 & 94,7 & 90,1 & 89,0 & $93,1^{*}$ & 89,5 & 86,4 & 87,3 \\
\hline & NR & 1,0 & 2,5 & 0,5 & 4,5 & 1,0 & $3,2^{*}$ & 0 & 1,2 \\
\hline
\end{tabular}

\begin{tabular}{|c|c|c|c|c|c|c|c|c|c|}
\hline \multirow{3}{*}{ 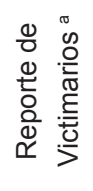 } & SI & 4,1 & 4,8 & 4,4 & $10,9^{*}$ & 4,3 & $11,3^{*}$ & 9,3 & $13,6^{*}$ \\
\hline & $\mathrm{NO}$ & $94,3^{*}$ & 90,2 & $93,4^{*}$ & 84,3 & 93,9 & 83,4 & $89,0^{*}$ & 82,0 \\
\hline & NR & 1,7 & $5,0^{*}$ & 2,3 & $4,8^{*}$ & 1,8 & $5,1^{*}$ & 1,7 & $4,4^{*}$ \\
\hline
\end{tabular}

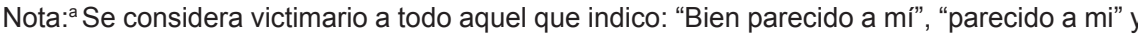

"un poco parecido a mi". ${ }^{b} \mathrm{~F}=$ Femenino, $\mathrm{M}=$ Masculino

${ }^{*} p<, 05$. 
Tabla 2. Prevalencia cyberbullying según Curso: Estudiantes víctimas y agresores

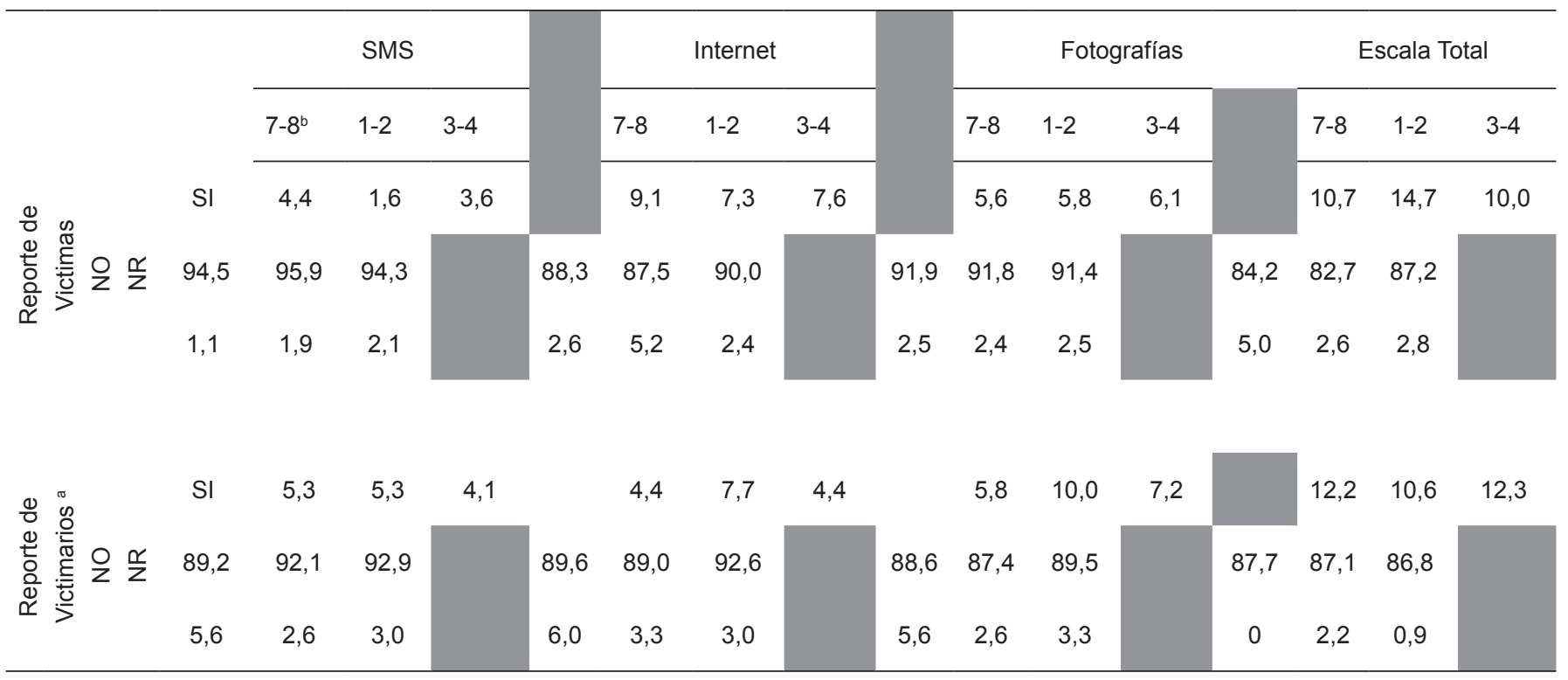

Nota: aSe considera victimario a todo aquel que indico: "Bien parecido a mí", "parecido a mi" y "un poco parecido a mi". b7-8= Séptimo y Octavo Básico, 1-2= Primero y Segundo Medio, 3-4= Tercer y Cuarto año de Enseñanza Media. Se realizaron contrastes de porcentajes entre todas las combinaciones posibles (7-8 vs. $1-2 ; 7-8$ vs. $3-4 ; 12$ vs. $3-4)$ sin encontrase ninguna diferencia estadísticamente significativa.

Tabla 3. Prevalencia cyberbullying según tipo de dependencia: Estudiantes víctimas y agresores.

\begin{tabular}{|c|c|c|c|c|c|c|c|c|c|c|c|c|c|c|c|c|}
\hline \multirow{5}{*}{$\frac{0}{0} \frac{O}{z}$} & & \multicolumn{3}{|c|}{ SMS } & & \multicolumn{3}{|c|}{ Internet } & & \multicolumn{4}{|c|}{ Fotografías } & \multicolumn{3}{|c|}{ Escala Total } \\
\hline & & $\mathrm{M}^{\mathrm{b}}$ & PS & $P$ & & M & PS & $P$ & & M & PS & $P$ & & M & PS & $P$ \\
\hline & SI & 4,3 & 3,5 & 2,9 & & 8,1 & 8,8 & 4,8 & & 5,5 & 7,6 & 5,0 & & 12,4 & 12,9 & 11,3 \\
\hline & 94,9 & 94,7 & 94,3 & & 89,6 & 88,4 & 93,0 & & 92,9 & 90,2 & 92,4 & & 87,6 & 86,6 & 86,6 & \\
\hline & 0,8 & 1,9 & 2,7 & & 2,3 & 2,8 & 2,2 & & 1,6 & 2,2 & 2,5 & & 0 & 0,5 & 2,1 & \\
\hline \multirow{3}{*}{ 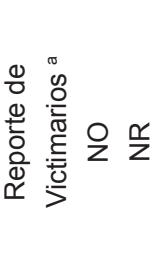 } & SI & 6,3 & 3,9 & 2,5 & & 7,5 & 7,5 & 9,3 & & 7,4 & 7,4 & 9,5 & & 9,9 & 11,7 & 13,2 \\
\hline & 90,3 & 92,2 & 95,7 & & 89 & 88,6 & 88,9 & & 89,4 & 88,5 & 88,9 & & 86,8 & 84,8 & 85,4 & \\
\hline & 3,3 & 3,8 & 1,7 & & 3,5 & 3,9 & 1,8 & & 3,2 & 4,1 & 1,6 & & 3,3 & 3,5 & 1,3 & \\
\hline
\end{tabular}


identificarse con el ejercicio de cyberbullying a través de internet $(z=3,90, p<, 05)$ y envío de fotografías a través del celular $(z=4,82, p<, 05)$ en forma más frecuente que las mujeres. Finalmente, la tasa de no respuesta de los hombres es mayor que la de las mujeres en cada uno de las estrategias de cyberbullying analizadas (SMS: $z=3,09, p<0,05$; Internet: $z=2,14, p<0,05$; Fotografías: $z=3,43, p<0,05$ )

\section{Prevalencia según Curso}

Los resultados indican que los estudiantes de los diferentes niveles escolares reportan haber sido víctima de cyberbullying en forma similar $(p \geq 05)$, tanto al considerar el cyberbullying en su conjunto (Escala Total, Tabla 2) como -al considerar- las diferentes modalidades a través del cual este es ejercido: envío de SMS, a través de Internet, y enviando fotos a través de los celulares. El mismo resultado se obtiene al considerar el reporte de los estudiantes respecto de la agresión a terceros través de las tecnologías $(p \geq 05)$.

\section{Prevalencia según Tipo de Dependencia}

Los resultados indican que los estudiantes que cursan estudios en establecimientos educacionales de diferentes tipos de dependencias reportan haber sido víctima de cyberbullying en forma similar ( $p \geq 05)$, tanto al considerar el cyberbullying en su conjunto (Escala Total, Tabla 3) como en cada una de las modalidades. Similar resultado se obtiene al considerar el reporte de los estudiantes respecto de la conducta de agredir $(p \geq, 05)$ en los diferentes tipos de establecimientos educacionales.

\section{Discusión}

Los resultados antes descritos reflejan la presencia de intimidación escolar cibernética o cyberbullying ya sea como victimario o agresor en valores que bordean el $12 \%$, es decir, aproximadamente 1 de cada 10 estudiantes participa de este fenómeno. Al comparar estos porcentajes con los reportados en la $3^{\circ}$ Encuesta de Nacional de violencia en el ámbito escolar 2009, se aprecia que el porcentaje de victimas es similar, sin embargo, en dicha encuesta solo un $6,4 \%$ de los estudiantes se sindicó como victimario por medio de internet (Ministerio del Interior \& Adimark Gfk, 2010).Parte de estas diferencias podrían obedecer a que en el presente estudio se consideró como universo solo aquellos estudiantes que tuvieran acceso a la tecnología, disminuyendo de esta forma el marco de muestra al cual se hace referencia, y develando de mejor manera la prevalencia real de los victimarios.

Si bien, el porcentaje encontrado es menor que el reportado en países desarrollados como Estados Unidos o Canadá (Li, 2006; Ybarra \& Mitchell, 2004) es superior al reportado en otros países hispanos, por ejemplo, en España solo un $5,5 \%$ de los estudiantes reconoce ser víctimas de cyberbullying (Defensor del Pueblo, 2007).
En forma similar a lo que ocurre con el bullying (Nansel et al., 2001), los adolescentes de género masculino es más frecuente que desarrollen conductas de cyberbullying, particularmente en los hombres es más frecuente que agredan a través de internet y/o envíen fotos no consensuadas a través del celular. Sin embargo, este patrón de género desaparece al considerar las víctimas de este fenómeno. Diferencias por género también se han evidenciado en estudios internacionales lo que implica entonces a tener en cuenta esta variable en futuros análisis (Li, 2006; Ybarra \&Mitchell, 2007).

En general, los resultados indican que la intimidación escolar cibernética o cyberbullying es un fenómeno que no solo afecta por igual a hombres y mujeres, sino que a jóvenes de diferentes edades y pertenecientes a diferentes contextos escolares. La homogeneidad en las víctimas se repite en los agresores al considerar las variables edad y contexto escolar. En contraste con la literatura antes expuesta, el presente estudio evidenció resultados similares a Patchin y Hinduja (2006), dónde no hay clara evidencia del rol de la edad como variable moderadora de la victimización de tipo cyberbullying.

El tipo de dependencia de los establecimientos educacionales da cuenta del contexto escolar y opera como un proxy de la realidad socio-económica que viven los estudiantes, contando con más recursos económicos aquellos pertenecientes a los establecimientos Particulares. Pese a que este grupo, en teoría tendría mayor acceso a las tecnologías, llama la atención que no se presenten diferencias según el tipo de establecimiento educacional. Esto podría entenderse a la luz del acceso cada vez menos inequiactivo a las tecnologías y su amplio uso en la juventud nacional (INJUV, 2010).

El presente estudio de carácter explorato es una primera aproximación descriptiva de este fenómeno en Chile y en la región. La demostración que el cyberbullying es un fenómeno existente en la realidad nacional, y que es -mayormente- homogéneo en presentación, posiciona una nueva pregunta, particularmente respecto a sus consecuencias en la salud escolar y el sistema educativo en general, tanto para víctimas como victimarios, que requieren de futuros estudios en Chile y en la región sobre el tema. Un especial foco de interés aparece también en torno al sistema escolar y su posible rol como agente preventivo, debido a que puede establecer y modelar relaciona es saludables de niños, niñas y adolescentes que asisten día a día a los establecimientos educacionales.

Teniendo en cuenta lo anterior, el estudio tiene algunas limitaciones que hay que considerar. Si bien los datos fueron ponderados para realizar los análisis, todavía corresponden a una ciudad del país, por ende cualquier generalización nacional debe tener en cuenta este posible sesgo. Por otra parte, las diferencias de género encontradas en el rol de victimario, deben ser también contextualizadas en las diferencias de género en las tasas de no respuesta, la cual fue mayor para los hombres. Futuras líneas de investigación, podrían seguir podrían seguir analizando con más detalle las 
diferencias de género en las dinámicas de cyberbullying en la escuela.

\section{Agradecimientos}

VTR, CRITEIRA Research y el CEEIN de la Univerisdad del Desarrollo

\section{Referencias}

Beale, A.,\& Hall, R. (2007). Cyberbullying: What school administrators (and parents) can do.The Clearing House,81, 8-12.doi: 10.3200/ TCHS.81.1.8-12.

Bond, L., Carlin, J., Thomas, L., Rubin, K.,\&Patton, G. (2001). Does bullying cause emotional problems? A prospective study of young teenagers. British Medical Journal, 323, 480-484.doi: 10.1136/ bmj.323.7311.480

Defensor del Pueblo (2007). Violencia escolar: el maltrato entre iguales en la Educación Secundaria Obligatoria 1999-2006. Madrid: Defensor del Pueblo.

Grills, A. \& Ollendick, T. (2002). Peer victimization, global selfworth, and anxiety in middle school children. Journal of Clinical Child and Adolescent Psychology, 31, 59-68.doi: 10.1207/153744202753441675

Hawker, D. \& Boulton, M. (2000). Twenty years research on peer victimization and psychosocial maladjustment: A meta-analytic review of cross-sectional studies. Journal of Child Psychology and Psychiatry, 41, 441-455.doi: 10.1111/1469-7610.00629

Hinduja, S. \& Patchin, J. W. (2009). Bullying beyond the schoolyard: Preventing and responding to cyberbullying. Thousand Oaks, CA: Corwin Press.

INJUV (2010). Sexta Encuesta Nacional de Juventud. Santiago, Chile: INJUV.

Kiriakidis, S. \& Kavoura, A. (2010). Cyberbullying: A review of the literature on harassment through the internet and other electronic means. Family \& Community Health, 33(2), 82-93.doi: 10.1097/ $\mathrm{FCH} .0 \mathrm{~b} 013 \mathrm{e} 3181 \mathrm{~d} 593 \mathrm{e} 4$

Kowalski, R. M., Limber, S. P. \& Agatston, P. W. (2012).Cyberbullying: Bullying in the digital age. Wiley-Blackwell.

Langos, C. (2012). Cyberbullying: the challenge to define. Cyberpsychology, Behavior and Social Networking, 15(6), 285289.doi:10.1089/cyber.2011.0588
Lecannelier, F., Varela, J., Astudillo, J., Rodríguez, J., \& Orellana, P. (2010). Adaptación y validación del instrumento "Internet Experiences Questionnaire" a escolares que cursan $7^{\circ}$ año básico a $4^{\circ}$ medio, de la ciudad de Santiago de Chile, en el año 2008. En: Paz Ciudadana Ed., Sexto Congreso Nacional de Investigación sobre Violencia y Delincuencia; 23-24 noviembre 2010; Santiago, Chile. Santiago: Fundación Paz Ciudadana; 2011.p.141-162. Recuperado de:http://www.pazciudadana.cl/ docs/pub_20110720132639.pdf

Li, Q. (2006). Cyberbullying in schools: A research of gender differences. School Psychology International, 27, 157-170. doi:10.1177/0143034306064547

Ministerio del Interior \& Adimark Gfk (2010). Tercera Encuesta nacional de Violencia en el Ámbito Escolar 2009. Santiago, Chile. Recuperado de:http://www.seguridadpublica.gov.cl/filesapp/ presentacion_violencia_escolar_2009_web.pdf

Nansel, T., Overpeck, M., Pilla, S., Ruan, J., Simons-Morton, B., \& Scheidt P. (2001). Bullying behaviors among US youth: Prevalence and association with psychosocial adjustment. The Journal of the American Medical Association, 285, 2094-2100.doi:10.1001/ jama.285.16.2094.

National Children's Home. (2002). NCH 2002 Survey. Recuperado de:http:www.nch.org.uk/itok/showquestion. asp?faq $=9 \&$ fldAuto $=145$

O'Moore, M., \& Kirkham, C. (2001). Self - Esteem and its relationship to bullying behaviour. Aggressive Behavior, 27, 269-283. doi: 10.1002/ab.1010.

Patchin, J. W. \& Hinduja, S. (2006). Bullies move beyond the schoolyard: A preliminary look at cyberbullying. Youth Violence and Juvenile Justice, 4(2), 148-169. doi:10.1177/1541204006286288

PNUD. (2006). Desarrollo Humano en Chile: Las nuevas tecnologías ¿Un salto al futuro? Santiago, Chile: PNUD.

Raskauskas, J., \& Stoltz, A. (2007). Involvement in traditional and electronic bullying among adolescents. Developmental Psychology, 43, 564-575.doi: 10.1037/0012-1649.43.3.564

Rigby, K. (2003). Consequences of bullying in schools. Canadian Journal of Psychiatry, 48, 583-590.

Salmon, G., James, A., \& Smith, D. (1998). Bullying in schools: self reported anxiety, depression, and self esteem in secondary school children. British Medical Journal,317, 924-925.

Smith, P. K., Mahdavi, J., Carvalho, M., Fisher, S., Russell, S., \& Tippett, N. (2008). Cyberbullying: its nature and impact in secondary school pupils. Journal of Child Psychology and Psychiatry, 49(4), 376-385. doi:10.1111/j.1469-7610.2007.01846.x 
Spears, B., Slee, P., Owens, L. \&Johnson, B. (2009). Behind the scenes and screens: Insights into the human dimension of covert and cyberbullying. Zeitschrift Für Psychologie, 217, 189-196.doi: 10.1027/0044-3409.217.4.189

Wong-Lo, M., Bullock, M., \& Gable, R. (2011). Cyber bullying: practices to face digital aggression. Emotional and Behavioural Difficulties, 16, 317-325. doi: 10.1080/13632752.2011.595098
Ybarra, M. \& Mitchell, J. (2004). Youth engaging in online harassment: Associations with caregiver-child relationships, internet use, and personal characteristics. Journal of Adolescence, 27, 319-336. doi:10.1016/j.adolescence.2004.03.007

Ybarra, M. L., \& Mitchell, K. J. (2007). Prevalence and frequency of Internet harassment instigation: implications for adolescent health. The Journal of adolescent health,41(2), 189-95. doi:10.1016/j. jadohealth.2007.03.005

Recebido em: 31/05/2013

Reformulado em: 15/08/2013

Aprovado em: 18/09/2013

\section{Sobre os autores}

Jorge Varela T. (jvarela@udd.cl). Psicólogo. Magíster en psicología educacional, Pontificia Universidad Católica de Chile. Docente Investigador Universidad del Desarrollo, Santiago de Chile.

J. Carola Pérez (janetperez@udd.cl) Psicóloga. Doctora en Psicología, Pontificia Universidad Católica de Chile. Centro de Estudios Evolutivos e Intervención en el Niño (CEEIN), Universidad del Desarrollo.

Hermann Schwaderer Z. (h.schwaderer@gmail.com) Sociólogo. Pontificia Universidad Católica de Chile. Fundación Paz Ciudadana.

Javiera Astudillo (jastudillob@udd.cl) psicóloga. Centro de Estudios Evolutivos e Intervención en el Niño (CEEIN), Universidad del Desarrollo.

Felipe Lecannelier A. (flecannelier@udd.cl) psicólogo. Magister en Filosofía, Universidad de Chile. Centro de Estudios Evolutivos e Intervención en el Niño (CEEIN), Univer 